\title{
Prevalence of rubella susceptibility among pregnant mothers in a community-based antenatal clinic in Malaysia: A cross-sectional study.
}

\begin{abstract}
Introduction: Routine rubella antibody screening is not done for antenatal mothers in community health clinics in Malaysia. However, congenital rubella syndrome has persisted with its associated health burden. Objectives: To determine the prevalence of rubella susceptibility among pregnant mothers and its associated risk factors. Methodology: A crosssectional study was carried out in the Petaling district, Selangor, Malaysia, where 500 pregnant mothers were recruited, and face-to-face interviews were conducted. Rubella IgG tests were performed. Results: The prevalence of rubella susceptibility among pregnant mothers was $11.4 \%$. Using logistic regression, a history of not having received rubella vaccination or having unknown rubella vaccination status was found to be a significant predictor for mothers to be rubella susceptible (odds ratio $=2.691 ; 95 \%$ confidence interval $=$ 1.539-4.207). Conclusions: Routine rubella IgG screening tests need to be offered to all antenatal mothers in view of the high prevalence found.
\end{abstract}

Keyword: Antenatal; Malaysia; Prevalence; Rubella susceptibility. 